\title{
Methylation of S100A8 is a promising diagnosis and prognostic marker in hepatocellular carcinoma
}

\author{
Kun Liu ${ }^{1,5, *}$, Yuening Zhang ${ }^{1, *}$, Chengdong Zhang ${ }^{1,6, *}$, Qinle Zhang ${ }^{2, *}$, Jiatong \\ $\mathrm{Li}^{1,}{ }^{*}$, Feifan Xiao ${ }^{1}$, Yingfang $\mathbf{L i}^{3}$, Ruoheng Zhang ${ }^{1}$, Dongwei Dou ${ }^{1}$, Jiezhen Liang ${ }^{4}$, \\ Jian Qin', Zhidi Lin ${ }^{1}$, Dong Zhao ${ }^{1}$, Min Jiang ${ }^{1}$, Zhenxin Liang ${ }^{1}$, Jie Su ${ }^{8}$, \\ Vanaparthy Pranay Gupta ${ }^{1}$, Min He ${ }^{7}$, Xiaoli Yang ${ }^{1}$ \\ ${ }^{1}$ Medical Scientific Research Center, Guangxi Medical University, Nanning, Guangxi 530021, China \\ ${ }^{2}$ Genetic and Metabolic Central Laboratory, The Maternal and Children Health Hospital of Guangxi, Guangxi 530002, China \\ ${ }^{3}$ Department of Endocrinology, Fourth Affiliated Hospital of Guangxi Medical University, Liuzhou, Guangxi, 545005, China \\ ${ }^{4}$ Department of Otolaryngology-Head and Neck Surgery, First Affiliated Hospital of Guangxi Medical University, Guangxi \\ 530021, China \\ ${ }^{5}$ Department of Gastroenterology, The Third Hospital of Nanchang City, Jiangxi 330009, China \\ ${ }^{6}$ Department of Microbiology and Microbial Engineering, School of Life Sciences, Fudan University, Shanghai 200438, China \\ ${ }^{7}$ School of Public Health, Guangxi Medical University, Guangxi 530021, China \\ ${ }^{8}$ Key Laboratory of Early Prevention and Treatment for Regional High Frequency, Ministry of Education, Guangxi Medical \\ University, Nanning, Guangxi 530021, China \\ *These authors have contributed equally to this work \\ Correspondence to: Xiaoli Yang, email: cncsyx|@126.com \\ Min He, email: hemin@gxmu.edu.cn
}

Keywords: hepatocellular carcinoma, S100A8, DNA methylation, pyrosequencing, prognosis Received: October 03, $2015 \quad$ Accepted: June 29, $2016 \quad$ Published: July 23, 2016

\section{ABSTRACT}

The abnormality of DNA methylation is one of the major epigenetic alterations in the human hepatocellular carcinoma (HCC). We have assessed the global genomic DNA methylation profiles in human HCC patients by using the Infinium Human Methylation27 BeadChip. A CpG loci of S100A8 was found to be significantly hypomethylated in HCC.

Pooled meta-analysis of five validation public datasets demonstrated its methylation level was significantly lower for HCC compared to paired adjacent normal tissues. Quantitative pyrosequencing analysis also showed that the S100A8 methylation level was decreased in cancer tissues $(31.90 \% \pm 13.31 \%)$ than that in the paired adjacent normal tissues $(65.33 \% \pm 3.64 \%, p<0.01)$. The area under the ROC curve (AUC) value was $0.950(p<0.01)$. Kaplan-Meier survival curves revealed that hypomethylation of S100A8 was associated with shortened overall survival (OS) and progression-free survival (PFS) (log rank $p<0.05)$. Multivariate Cox proportional hazards model also indicated significantly shorter OS (HR, 1.709; $95 \%$ CI, 1.127-2.591) and PFS (HR, 1.767; $95 \%$ CI, 1.168-2.974) were observed in the low-methylation-level group compared to the high-methylation-level group. Furthermore, S100A8 overexpression in Huh7 and MHCC-97H hepatoma cell lines led to increased cell proliferation, migration, invasion, and tumor growth. These findings suggested S100A8 methylation to be served as potential diagnosis and prognosis marker for HCC. S100A8 also may play as a tumor promoter in HCC.

\section{INTRODUCTION}

Hepatocellular carcinoma (HCC) is one of the most common and aggressive tumors. Not only its incidence expands in the past decades, $\mathrm{HCC}$ is also a leading cause of cancer-related deaths worldwide [1-4]. Although considerable efforts that have been made in the treatment of HCC such as surgical resection, liver transplantation 
and chemotherapy, the mortality rate remains high, and it is largely due to relapse after surgery or formation of intra-hepatic metastases [5-7]. Given the fact that most HCC patients at the advanced stage are prone to present a poor prognosis, there is an urge for the identification of effective diagnostic and prognostic biomarkers for HCC. Currently, the serum AFP test is widely used, however, with a relatively low sensitivity, thus its application is barely satisfactory and its clinical value is limited [8].

Aberrant DNA methylation is a common event during the pathogenesis of human cancers and one of the important epigenetic mechanisms in carcinogenesis. Loss of methylation primarily affects repetitive genomic elements and gene bodies, while hypermethylation mostly occurs at the promoters of tumor suppressor genes [9-12]. Many studies have provided evidence that cancer-linked DNA methylation alterations may be used as early indicators of HCC, as well as prognostic markers of cancer progression and response to chemotherapy $[13,14]$. Until recently, mounting evidence indicates that the hypomethylation of "normally" methylated genes are significant in the pathogenesis of HCC. A number of hypomethylated tumor-promoting genes, including LINE1, DNC, HPA, TFF3, MAT2A, HKII, CD147 and VIM have been identified in primary human $\mathrm{HCC}$ [15-18].

S100A8, also known as calcyclin, is a lowmolecular-weight calcium-binding protein which belongs to the S100 family. S100A8 is involved in the regulation of a wide range of cellular processes, such as cell proliferation, the dynamics of cytoskeleton components, differentiation, $\mathrm{Ca}^{2+}$ homeostasis, and apoptosis $[19,20]$. The expression of S100A8 is in a cell-specific manner, mainly in fibroblasts and epithelial cells [21, 22]. Furthermore, high levels of S100A8 have been found in some diseases, especially in cancer. So far the protein level of S100A8 has been studied by immunohistochemistry and shown to be up-regulated in colorectal carcinoma, lung cancer, pancreatic cancer, breast cancer and thyroid carcinoma [23-30]. Expression of several members of the S100 family, including S100A2, S100A4, S100A6, and $\mathrm{S} 100 \mathrm{P}$, are known to be regulated epigenetically. The declining expression of S100A8/A9 in HNSCC patients is associated with increased DNA methylation [31]. DNA demethylation resulted in S100A8 increased in myeloid cells [32]. There was rare study of S100A8 methylation in $\mathrm{HCC}$.

In the present study, we analyzed the paired samples of tissues from the HCC patients to find a novel methylation alteration through genome-wide DNA methylation array screening and meta-analysis. After that, pyrosequencing was used to quantify the methylation levels of S100A8 in HCC samples, and the diagnostic value of S100A 8 was examined. We further explored the potential role of S100A8 methylation in HCC prognosis using TCGA microarray datasets. At last, the function of S100A8 in HCC cell lines were investigated.

\section{RESULTS}

\section{Methylation microarrays}

Global DNA methylation profiles were measured by Illumina Infinium Human Methylation27 BeadChips, which target 14,475 total refseq genes, 12,833 wellannotated genes described in the NCBI CCDS database, 144 methylation hotspots in cancer genes, 982 cancerrelated targets and 110 miRNA promoters. We performed genome-wide methylation profiling with $3 \mathrm{HCC}$ tissues and their adjacent normal tissues. Two methylation sites of S100A8 were detected (Figure 1A and Table 1). As shown in Figure 1B, the CpG site ( $\operatorname{cg} 20070090)$ of S100A8 is hypomethylated in three pairs of tissues $(p<0.01)$, while the CpG site (cg24898863) is not changed significantly.

\section{Meta-analysis of five HCC methylation expression datasets identifies hypomethylation of S100A8}

We identified and manually curated five published and publicly available HCC datasets. Only datasets containing both $\mathrm{HCC}$ and their adjacent normal samples were used for further analysis. Four datasets from GEO and part of the TCGA dataset finally met this criteria (246 HCC, 246 adjacent normal; Supplementary Table S1). A meta-analysis approach was applied to these datasets as outlined in Figure 2A. As expected, the site (cg20070090) is hypomethylated significantly in the validation datasets. Geometric mean of the cg20070090 was significantly higher for HCC compared to normal samples in each of the five validation datasets.

\section{Verification of S100A8 methylation by pyrosequencing}

We then confirmed the methylation alterations of S100A8 in 52 HCC tissue samples and their adjacent tissues by a highly quantitative method using Pyrosequencing ${ }^{\mathrm{TM}}$ technology.

For the site of cg20070090, the average DNA methylation levels were significantly decreased $(\mathrm{p}<0.01)$ in HCC tissues $(32.93 \% \pm 14.82 \%)$ than that in their paired controls $(64.91 \% \pm 4.65 \%)$ (Figure $3 \mathrm{C})$.

\section{ROC curve analysis of S100A8 methylation}

To further evaluate the diagnostic significance of S100A8, we constructed a receiver operating characteristic (ROC) curve by plotting the sensitivity versus the specificity (Figure 3D). The area under the ROC curve (AUC) is $0.950(p<0.01)$. Based on the maximum Youden index discriminating patients with HCC from controls, an optimal cutoff value of $54.025 \%$ is obtained and its sensitivity and specificity for predicting HCC is $86.53 \%$ 
and $98.07 \%$, respectively. Our results demonstrated the methylation level of the majority of tumor tissues was less than the cutoff value.

\section{Association between S100A8 methylation level and clinical, epidemiological, and pathological variables}

We analyzed the relationship between the S100A8 methylation level in HCC and various clinical, epidemiological, and pathological variables. However, none of the clinical HCC features was significantly correlated with S100A8 methylation level (Table 2).

\section{S100A8 methylation level and patient survival}

We assessed the association of the S100A8 methylation level with survival outcomes. Among 345 patients, 121 of them were dead within a median follow-up of 1.997 years. Based on methylation level of tumor samples, patients were divided into four quartiles (Q1: <25\%; Q2: 25\%-49\%; Q3: 50\%-74\%; Q4: $\geq 75 \%$, Supplementary Table S2). The results of Kaplan-Meier analysis showed that OS and PFS were both significantly different among the four groups (Estimate Median OS: Q1:2.753; Q2:4.274; Q3:5.074; Q4:8.926, log-rank $p<0.05$. Estimate Median PFS: Q1:2.266; Q2:3.096;

A

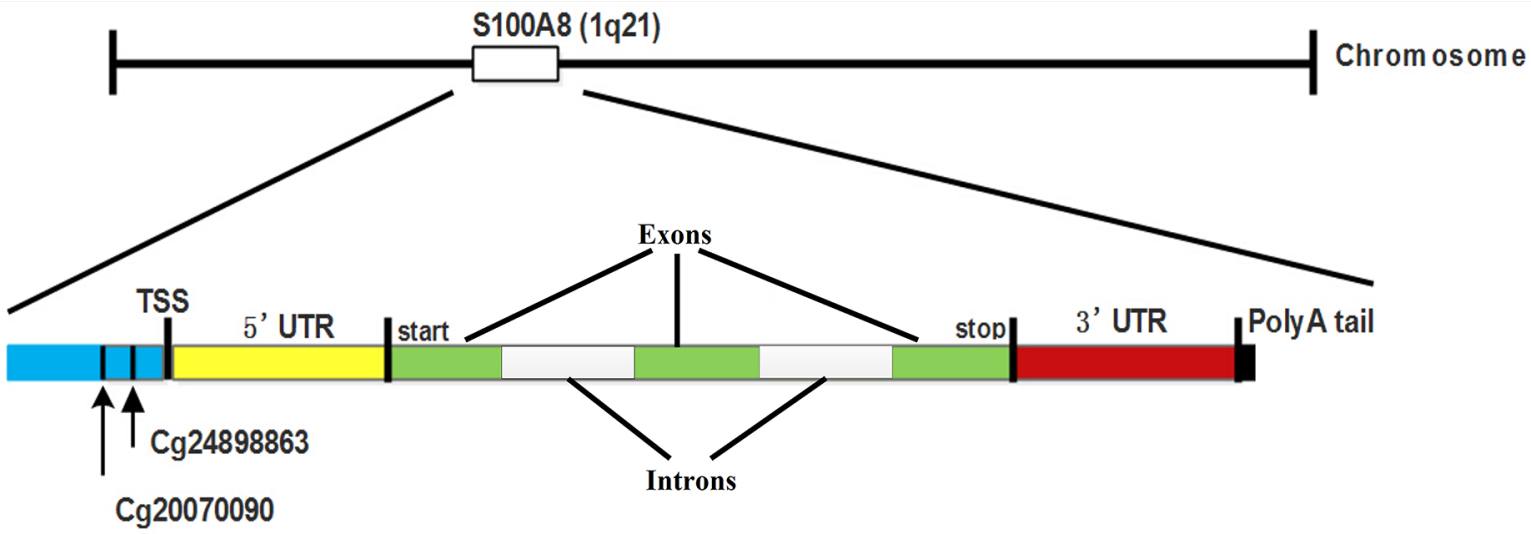

B

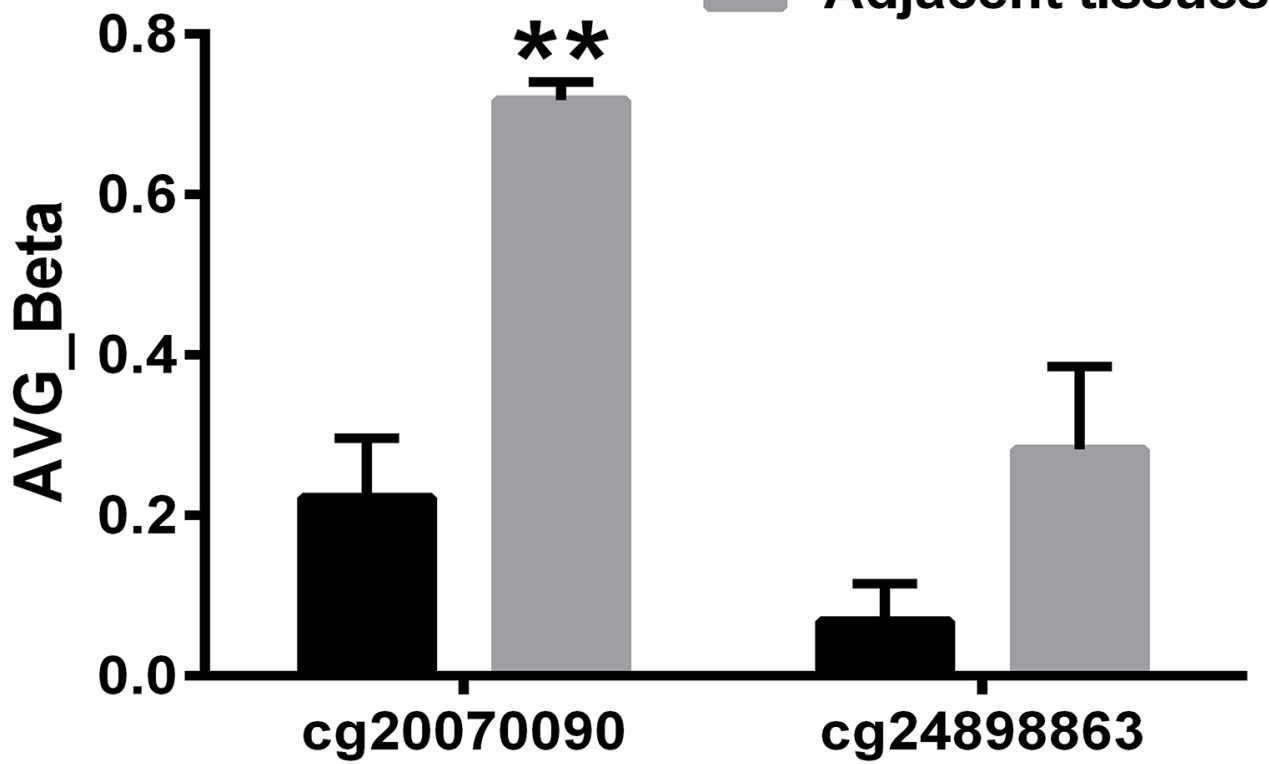

Figure 1: Gene structure of S100A8. A. S100A8 gene is located in the 1q21 chromosome. S100A8 consists of three exons and two introns. The two methylation sites of the gene are upstream of transcript start site. B. Average Beta of methylation sites in S100A8. Test sites are listed on the $\mathrm{x}$ axis, and the mean methylation level is shown on the y axis. Significant differences $(p<0.01)$ are denoted with two asterisks. 
Table 1: Sequence information of two methylation sites of the S100A8

\begin{tabular}{|c|c|c|c|c|}
\hline Target ID & Symbol & Sequence & CpG island & Distance to TSS \\
\hline cg20070090 & S100A8 & $\begin{array}{l}\text { TCTCCTCTCTCAGGAA } \\
\text { GGCTGCTCCACTTCCCTG } \\
\text { ACCCTCCCCAAGAGAAGCCC } \\
\text { AAAGTG[CG]GGGCCA } \\
\text { ACCCAGACAGTCCCA } \\
\text { CTTACCAGGTCTTCTGAAA } \\
\text { GACAGCTGACAAGAGACATG }\end{array}$ & FALSE & 60 \\
\hline $\operatorname{cg} 24898863$ & S100A8 & $\begin{array}{l}\text { GTCTTCTGAAAGACAG } \\
\text { CTGACAAGAGACATGCAGG } \\
\text { GCTGAGAGGCAGCTC } \\
\text { CTTTTTATAG[CG]GTTA } \\
\text { GGCTTGGCCAGCTGCC } \\
\text { CACAGCTTCAG } \\
\text { GCCATCAGAGACAGC } \\
\text { TTCTCCCTGCCAGA }\end{array}$ & FALSE & 31 \\
\hline
\end{tabular}

Q3:3.981; Q4:8.926. log-rank, $p<0.05)$. Q1 was defined as the 'low-methylation-level group' and Q2, Q3, and Q4 were combined into the 'high-methylation-level-group'. The 'low-methylation-level group' was significantly shorter than the 'high-methylation-level-group' for OS and PFS (Estimate Median OS time: Q1:2.753; Q2-4:4.907, log-rank $p<0.05$. Estimate Median PFS time Q1:2.266; Q2-4:3.367, log-rank $p<0.05$ ) (Figure 4, Table 3). Furthermore, according to the results of the multivariate Cox proportional hazards model, OS and PFS were significantly shorter in the 'low-methylation-level-group' than in the 'high-methylation-level group'. The HRs for OS and PFS were 1.709 (95\% CI 1.127-2.591, $p=0.032)$ and 1.767 (95\% CI 1.168-2.974, $p=0.037)$, respectively.

\section{Overexpressed S100A8 promotes cell proliferation, migration, invasion, and tumor growth in cultured Huh7 and MHCC-97H liver cancer cell lines}

To assess the role of S100A8 in HCC, we established S100A8-transfected Huh7 and MHCC-97H cells by a lentiviral system with puromycin selection. After puromycin selection, S100A8 expression in Huh7 and MHCC-97H cells was measured by western blot analysis and qPCR (Figure 5A and 5B). S100A8 overexpression promoted the cell viability of Huh7 and $\mathrm{MHCC}-97 \mathrm{H}$ compared to that of the empty vector control (Figure 5C). We performed a Scratch-wound assay and cell invasion assay to evaluate the effect of S100A8 on cell migration and cell invasion. The result showed that overexpressed S100A8 significantly promoted the migration of Huh7 cells and invasion of MHCC-97H cells respectively. (Figure 5D and 5E). We also examined the effect of S100A8 on tumorigenicity in vivo using subcutaneously tumor model in nude mice. Compared to the control group,
S100A8 overexpression resulted in significant promotion of tumor growth (Figure 5F)

\section{DISCUSSION}

Methylation alterations of tumor-associated genes are frequently observed in the development of cancer and may occur at different stages of HCC [33-37]. We used the genome-wide methylation array to screen the HCCspecific methylation alterations, and found methylation level of the site $(\operatorname{cg} 2007009)$ in S100A8 decreased significantly in HCC. Our result was confirmed by the meta-analysis of 5 publicly available methylation datasets, which greatly improved the robustness and credibility of our finding. Furthermore, we assessed the value of S100A8 methylation in HCC diagnosis, AUC was 0.950, which is higher than $\operatorname{AFP}(0.902)$, vascular endothelial growth factor (VEGF) (0.841) and serum leptin (Lep) (0.712) [38]. Although it still needs to be verified by a large amount of data and clinical experiments, our data certainly supported a potential role for S100A8 hypomethylation as a diagnostic $\mathrm{HCC}$ biomarker.

As is known to all, the prognosis of $\mathrm{HCC}$ is related to the patient's age, gender, liver function, tumor metastasis, pathological changes, the level of AFP, the activity of immune cells, the number of platelets, and so on [39-41]. However, there are few reports about the relationship between the methylation level of S100A8 and the prognosis of liver cancer. After analyzing the samples with clinic information in TCGA database, we found that the average OS and PFS of the HCC group with a relatively higher degree of methylation had a longer survival time than that of lower degree group. The patients with lower S100A8 methylation level showed poorer PFS and OS rates compared with S100A8 high-level group. This may indicate that one site of S100A8 methylation 


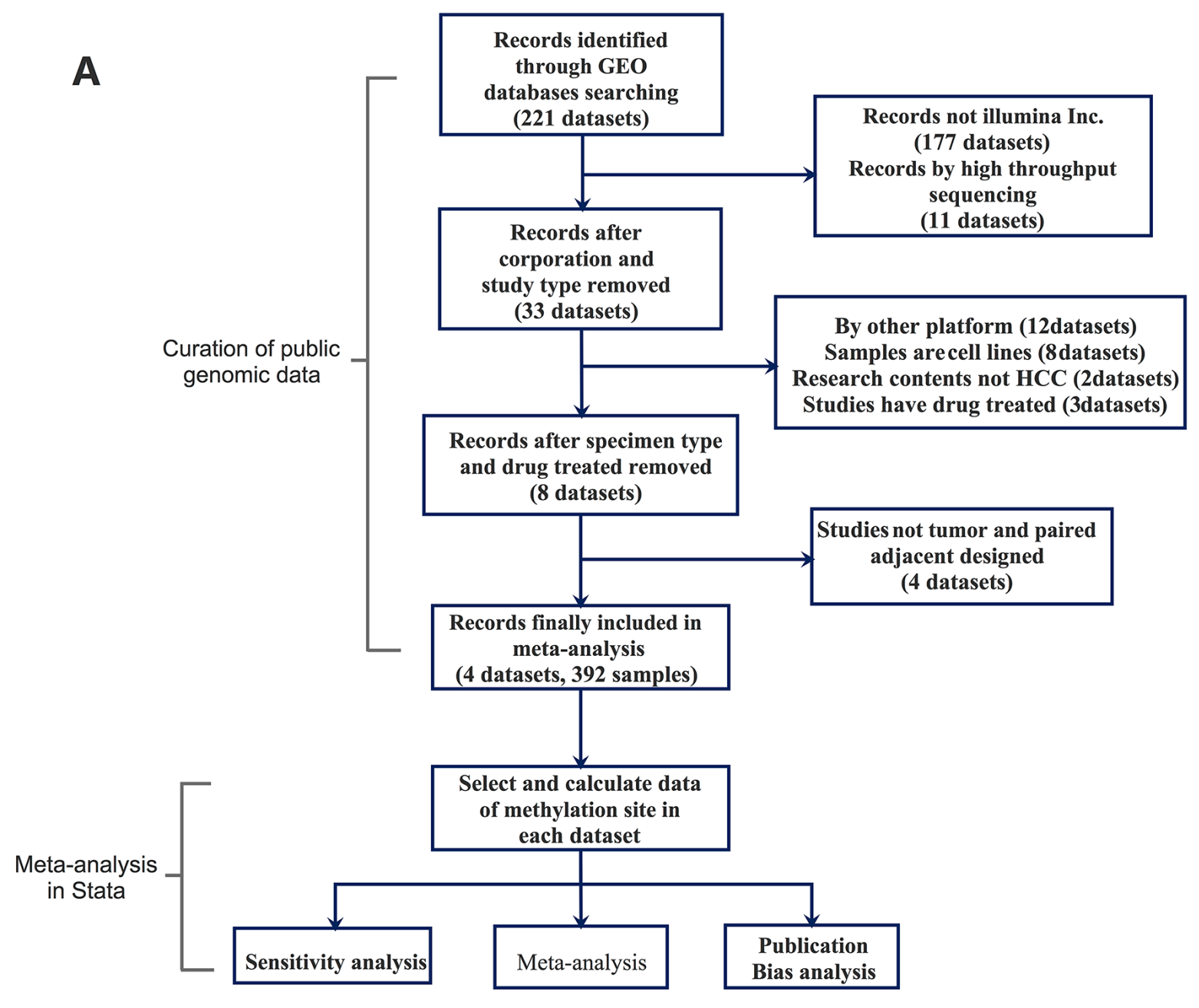

B

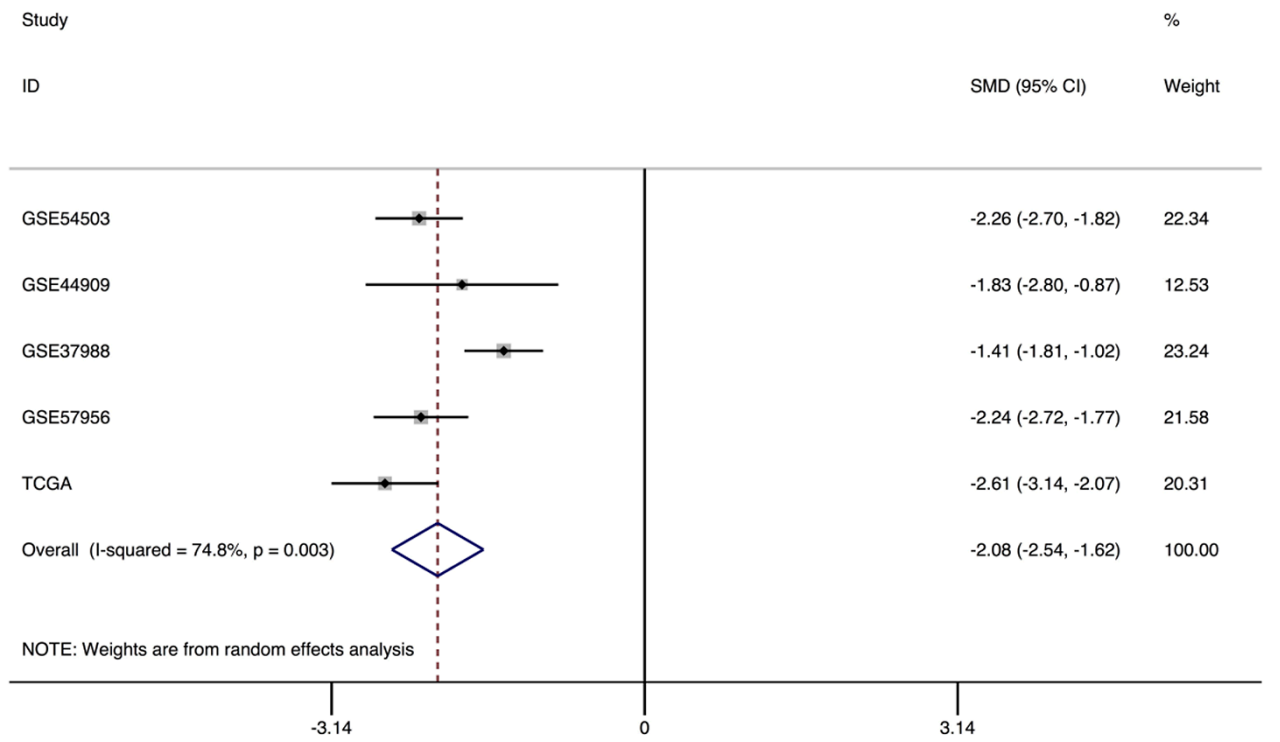

Figure 2: Meta-analysis of methylation cite of S100A8. A. Flow chart of Meta-analysis. B. Forest plot for the association between S100A8 Methylation and HCC using a random-effect model. SMD: standard mean difference (tumor vs. adjacent normal tissue). 
level can be used as a molecular biomarker for the prognosis of HCC. However, due to the lack of survival time in our data, a large well designed cohort is warranted to get a more precisely prediction result in China.

S100A8 is involved in the regulation of a wide range of cellular processes, such as cell proliferation, the dynamics of cytoskeleton components, differentiation, $\mathrm{Ca}^{2+}$ homeostasis, and apoptosis [19, 20]. Interestingly, S100A8 has been found to be upregulated incolorectal carcinoma, lung cancer, pancreatic cancer, breast cancer and thyroid carcinoma [23-30]. S100A8 ablation in mice can decrease cell proliferation, leading to significant reduction of tumor size [42]. S100A8 treatment can increase the viability and migration of colorectal carcinoma cells [43].We performed a series of experiments to evaluate the role of S100A8 in HCC. The results showed that the $\mathrm{S} 100 \mathrm{~A} 8$ overexpression in Huh7 and MHCC-97H cells induced marked increase in cell proliferation, migration, invasion, and tumor growth. These results suggested that $\mathrm{S} 100 \mathrm{~A} 8$ might be a tumor promoter in HCC cells.

In summary, our data demonstrated that S100A8 were frequently hypermethylated in non-tumor tissues but hypomethylated in HCC tissues. The AUC is higher than other general biomarkers of HCC. Hypomethylation of S100A8 was associated with both a shortened PFS and OS.
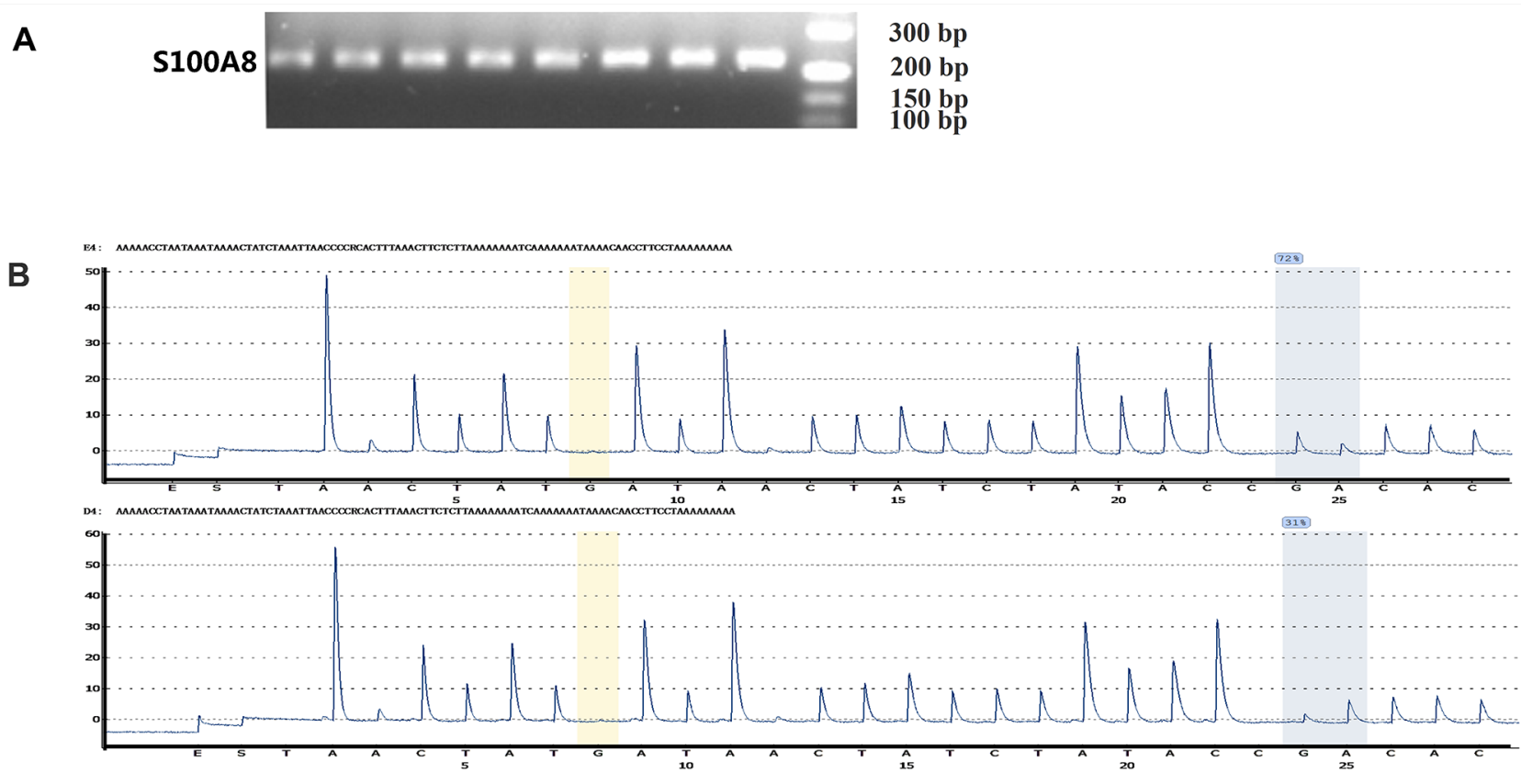

C

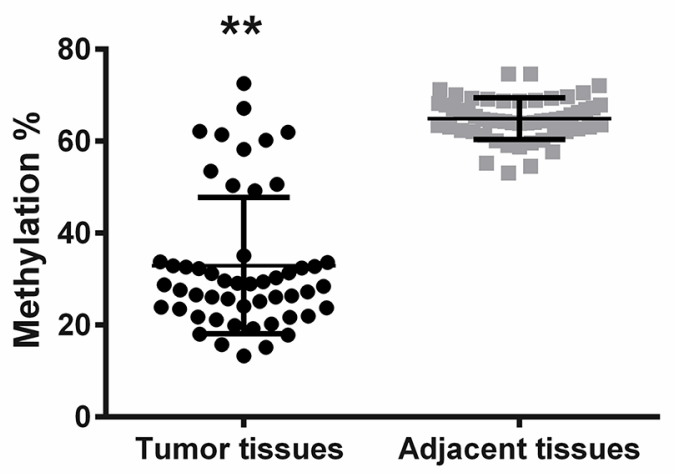

D

ROC Curve

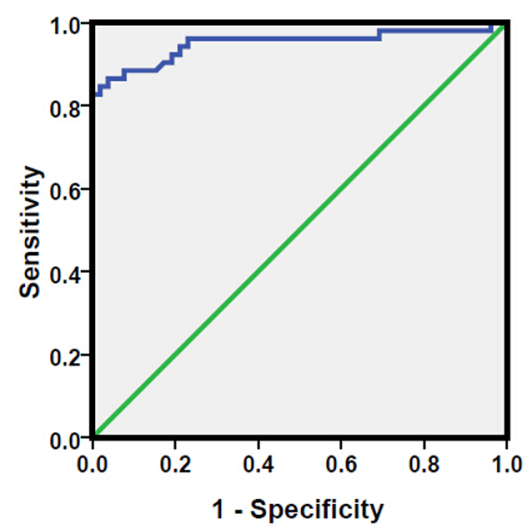

Figure 3: Methylation lever analysis of S100A8. A. Methylation-specific PCR of the S100A8 gene. Amplification of bisulfitetreated the target DNA. Methylated PCR products are shown as a $226 \mathrm{bp}$ band. B. Pyrosequencing was using to analyse the methylation lever. The methylation density of pyrosequencing is presented in two Pyrogram ${ }^{\circledR}$ charts as the methylation level of the CpG sites analyzed. C. Pyrosequencing analysis of the special site of the gene's methylation level in HCC samples and non-tumor samples. Significant differences were observed for S100A8 between tumor tissues and adjacent tissues. Vertical line denotes the grand mean expression value in both groups. D. ROC Curve. AUC of S100A8 was calculated to discriminate HCC tissues and the corresponding non-tumor tissues. 
Table 2: Status of S100A8 methylation in HCC tissues, and their relations to clinical and tumor features

\begin{tabular}{|c|c|c|c|}
\hline Clinic pathological & Total cases & $\begin{array}{l}\text { S100A8 methylation lever } \\
(\%)[\text { mean } \pm \text { SD }]\end{array}$ & p-value \\
\hline Gender & & & $>0.05$ \\
\hline male & 47 & $32.88 \pm 15.17$ & \\
\hline female & 5 & $33.79 \pm 8.73$ & \\
\hline Age & & & $>0.05$ \\
\hline$>66$ years old & 7 & $40.40 \pm 19.22$ & \\
\hline$\leq 66$ years old & 45 & $31.77 \pm 13.48$ & \\
\hline Smoking history & & & $>0.05$ \\
\hline Yes & 13 & $36.58 \pm 15.99$ & \\
\hline No & 39 & $31.17 \pm 12.18$ & \\
\hline Drinking history & & & $>0.05$ \\
\hline Yes & 14 & $34.86 \pm 17.49$ & \\
\hline no & 38 & $32.22 \pm 13.43$ & \\
\hline Serum HBV antigen & & & $>0.05$ \\
\hline positive & 30 & $30,80 \pm 13.23$ & \\
\hline negative & 22 & $35.83 \pm 16.00$ & \\
\hline The AFP in serum & & & $>0.05$ \\
\hline$>500 \mathrm{ng} / \mathrm{ml}$ & 31 & $32.06 \pm 13.80$ & \\
\hline$\leq 500 \mathrm{ng} / \mathrm{ml}$ & 21 & $34.21 \pm 15.80$ & \\
\hline Diameter of tumor & & & $>0.05$ \\
\hline$>5 \mathrm{~cm}$ & 37 & $34.16 \pm 15.39$ & \\
\hline$\leq 5 \mathrm{~cm}$ & 15 & $29.89 \pm 12.24$ & \\
\hline Portal vein & & & $>0.05$ \\
\hline yes & 9 & $27.00 \pm 6.73$ & \\
\hline no & 43 & $34.17 \pm 15.56$ & \\
\hline Metastasis & & & $>0.05$ \\
\hline yes & 5 & $27.37 \pm 12.30$ & \\
\hline no & 47 & $33.52 \pm 14.80$ & \\
\hline Child-Pugh classification & & & $>0.05$ \\
\hline A & 2 & $49.91 \pm 17.25$ & \\
\hline B & 39 & $34.00 \pm 15.18$ & \\
\hline $\mathrm{C}$ & 11 & $24.04 \pm 6.63$ & \\
\hline Differentiation & & & $>0.05$ \\
\hline Moderate-Poor & 17 & $38.80 \pm 17.73$ & \\
\hline Well-defined & 35 & $30.08 \pm 12.63$ & \\
\hline
\end{tabular}

The results of this study indicate that detection of S100A8 methylation level would be helpful to diagnose HCC and predict HCC patient prognosis. Methylation of S100A8 is a promising diagnosis and prognostic marker in HCC. Moreover, we demonstrated that S100A8 was probably a tumor promoter of HCC. 
A
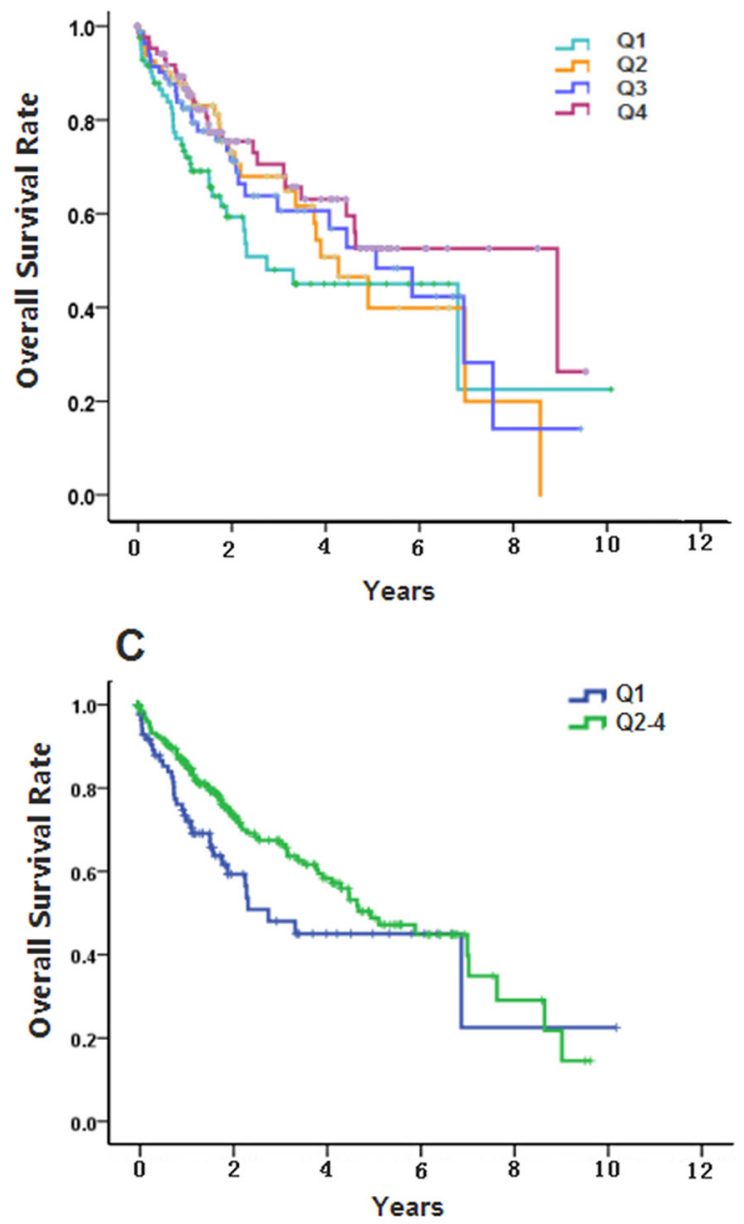

B
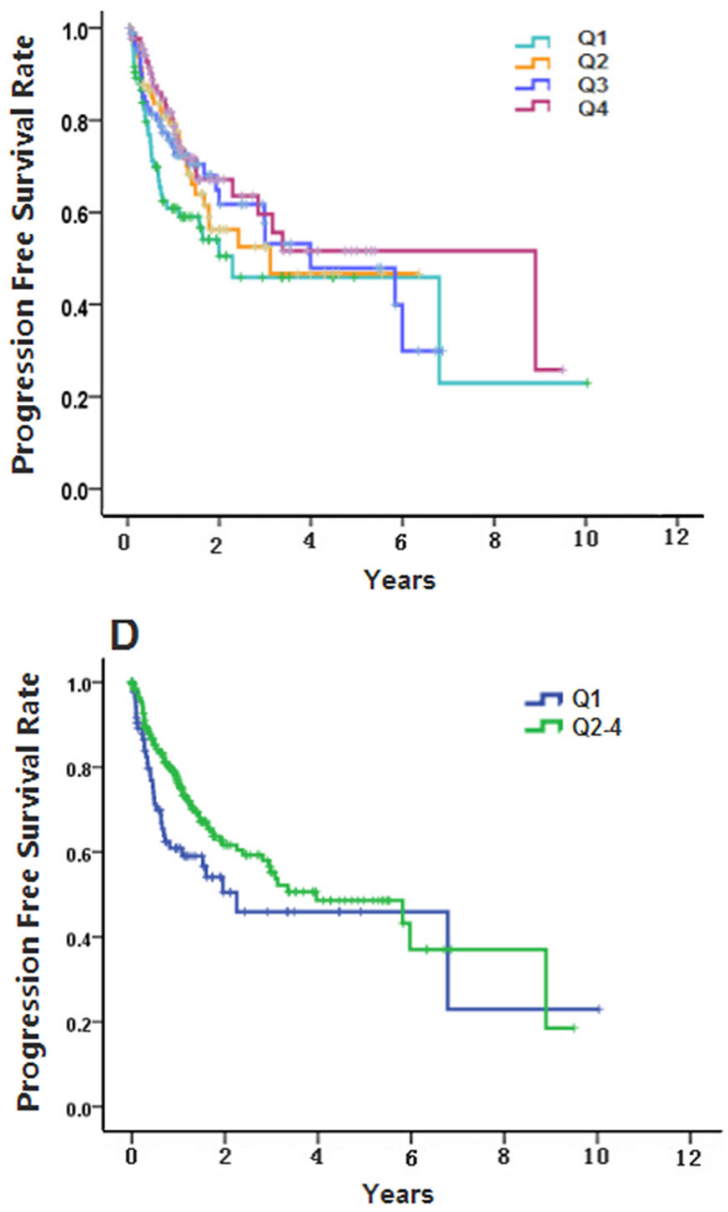

Figure 4: Kaplan-Meier curves of overall survival (OS) and progression-free survival (PFS) of different quartiles of S100A8 methylation level in 345 hepatocellular carcinomas. A. Kaplan-Meier analysis showed that OS were significantly different among the Q1, Q2, Q3 and Q4 groups (log-rank $p<0.05$ ). B. PFS were significantly different among the Q1, Q2, Q3 and Q4 groups $(\log$-rank $p<0.05)$. C. OS of Q1 group was significantly longer than the Q2-4 group (log-rank $p<0.05)$. D. PFS of Q1 group was significantly longer than the Q2-4 group (log-rank $p<0.05)$.

Table 3: Medians (years) for OS and PFS of four groups

\begin{tabular}{|c|c|c|c|c|c|c|c|c|}
\hline \multirow[t]{3}{*}{ group } & \multicolumn{4}{|c|}{ OS } & \multicolumn{4}{|c|}{ PFS } \\
\hline & \multirow[t]{2}{*}{ Estimate } & \multirow{2}{*}{$\begin{array}{l}\text { Std. } \\
\text { Error }\end{array}$} & \multicolumn{2}{|c|}{ 95\% Confidence Interval } & \multirow[t]{2}{*}{ Estimate } & \multirow{2}{*}{$\begin{array}{l}\text { Std. } \\
\text { Error }\end{array}$} & \multicolumn{2}{|c|}{ 95\% Confidence Interval } \\
\hline & & & $\begin{array}{l}\text { Lower } \\
\text { Bound }\end{array}$ & $\begin{array}{l}\text { Upper } \\
\text { Bound }\end{array}$ & & & $\begin{array}{l}\text { Lower } \\
\text { Bound }\end{array}$ & Upper Bound \\
\hline Q1 & 2.753 & 0.949 & 0.894 & 4.613 & 2.266 & 1.191 & 0 & 4.601 \\
\hline Q2 & 4.274 & 0.541 & 3.214 & 5.334 & 3.096 & . & . & . \\
\hline Q3 & 5.074 & 1.019 & 3.076 & 7.072 & 3.981 & 1.431 & 1.176 & 6.785 \\
\hline Q4 & 8.926 & 2.748 & 3.54 & 14.312 & 8.926 & 3.63 & 1.812 & 16.04 \\
\hline Q2-4 & 4.907 & 0.684 & 3.566 & 6.247 & 3.981 & 1.181 & 1.667 & 6.295 \\
\hline Overall & 4.616 & 0.711 & 3.222 & 6.011 & 3.367 & 1.039 & 1.33 & 5.404 \\
\hline
\end{tabular}


A
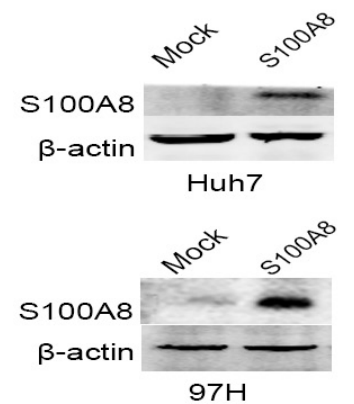

C
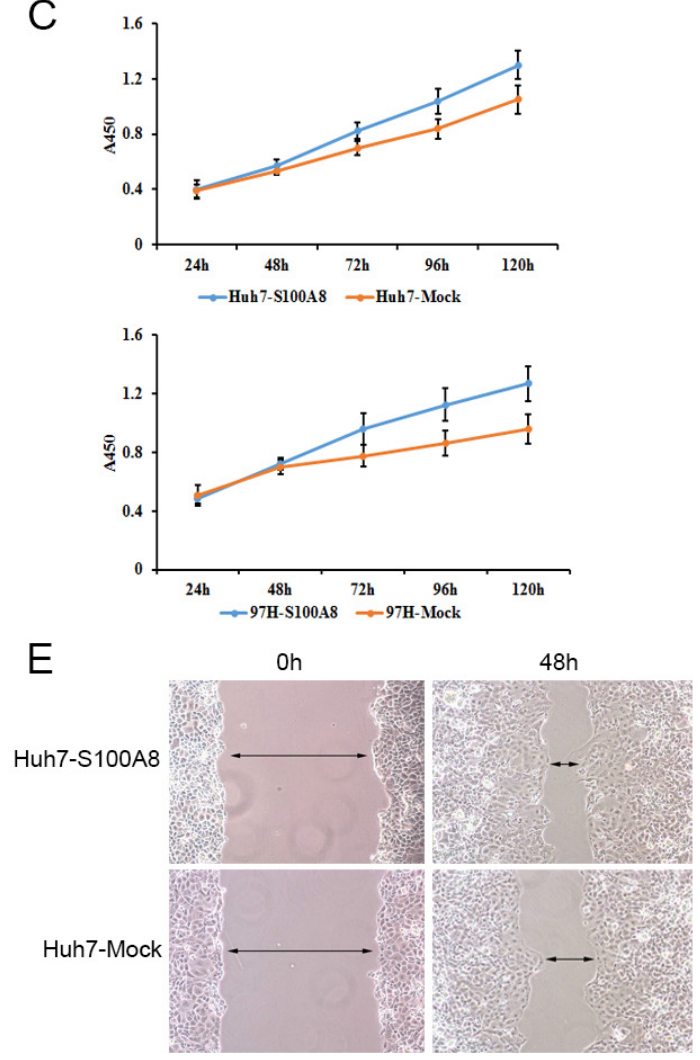

B

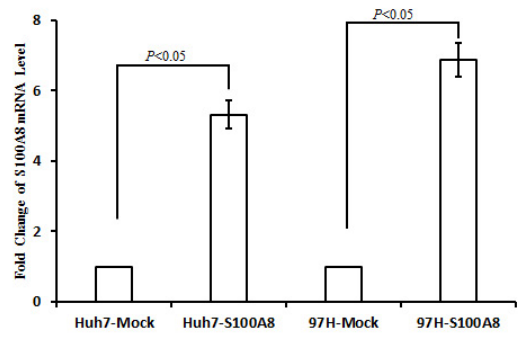

$\mathrm{D}$

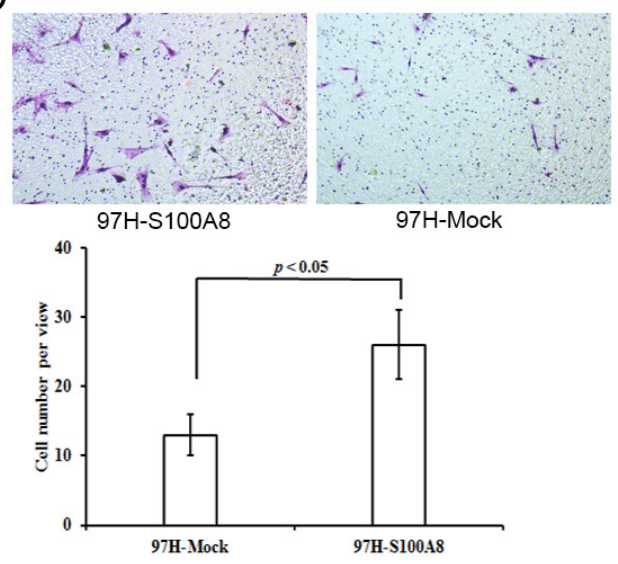

$\mathrm{F}$

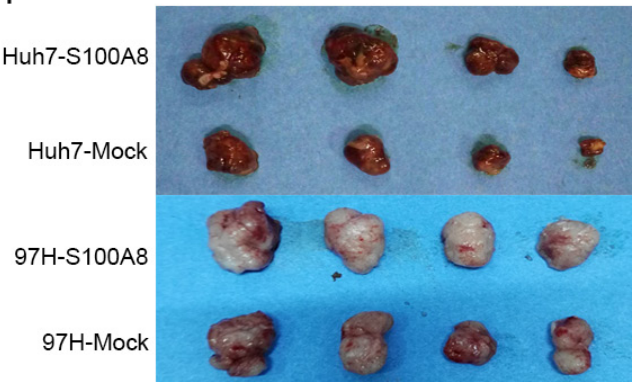

Figure 5: The functional importance of S100A8 in Huh7 and MHCC-97H hepatocellular carcinoma cells. A-B. Western blot analysis and qPCR were used to detect S100A8 expression in S100A8-transfected Huh7 and MHCC-97H cell lines. C. On the basis of CCK8 analysis, S100A8 overexpression in Huh7 and MHCC-97H could inhibit cell proliferation (data are shown as averages \pm SD, $\mathrm{n}=5$ ). D. Scratch-wound assay showed that S100A8 overexpression in Huh7 could promote cell migration. E. Overexpression of S100A8 in MHCC-97H could increase cell invasion (data are shown as averages $\pm \mathrm{SD}, \mathrm{n}=3$ ). F. Overexpression of S100A8 in Huh7 and MHCC$97 \mathrm{H}$ could promote tumor growth in nude mice.

\section{MATERIALS AND METHODS}

\section{Cell lines}

HCC cell line Huh7 was purchased from the Chinese Academy of Sciences, Shanghai, China. HCC cell line MHCC-97H was established at the Liver Cancer Institute, Zhongshan Hospital, Fudan University. The cells were maintained in DMEM supplemented with $10 \%$ fetal calf serum. The cells were incubated at $37^{\circ} \mathrm{C}$ in a humidified chamber containing $5 \% \mathrm{CO}_{2}$.

\section{Patients and tissue samples}

HCC tumor tissue samples and paired adjacent normal tissue samples were obtained by hepatectomy from the First Affiliated Hospital of Guangxi Medical University, following written informed consent and approved by the ethics committee. All tumors were classified according to the International Union Against Cancer tumor-node-metastasis classification system and World Health Organization criteria. Clinic pathologic data for parameters such as patient's age, gender, original 
tumor size, infection of HBV, serum level of AFP, portal vein tumor embolus, and degree of differentiation were collected. All tissue samples were stored in liquid nitrogen.

\section{DNA isolation and bisulfite conversion}

DNA was exacted by a QIAamp DNA Mini Kit (Germantown, MD, USA). Genomic DNA was then bisulfite-converted with an EpiTect Bisulfite Kit (QIAGEN) according to manufacturer's protocol.

\section{Methylation microarrays}

Bisulfite-converted genomic DNA was analyzed by The Infinium Methylation-27 Assay. Chip processes were performed by using reagents provided in the kits and following manufacturer's manual. Data were extracted and summarized using GenomeStudio software. Arrays that did not pass quality control in terms of b-distriutions and expected p-values across the arrays were removed. Methylation scores represented as $\beta$ values were generated for each site using Illumina Genome Studio Methylation module v1.8 (Illumina Inc., USA) and were computed based on the ratio of methylated to methylated plus ummethylated signal outputs. Control panel in the BeadStudio analytical software showed excellent intensity for staining, clear clustering for the hybridization probes, good target removal intensity and satisfactory bisulfite conversion [44].

\section{Data collection from public databases}

Four methylation $\begin{gathered}\text { datasets } \\ \text { (accession }\end{gathered}$
numbers GSE54503, GSE44909, GSE37988,
GSE57956) generated by the Illumina Infinium
HumanMethylation27K or 450K platforms were
downloaded from the NCBI GEO database. Background
correction was performed by Illumina GenomeStudio
software, which were declared by the data contributor
in GEO database. And for GSE54503 dataset, we also
performed the beta-mixture quantile normalization
(BMIQ) [45]. Furthermore, we downloaded DNA
methylation data with clinical data of HCC from The
Cancer Genome Atlas (TCGA). Data processing was
performed using RnBeads software. We thus use the
same background correction-method with GenomeStudio
software and BMIQ method were used for the
normalization process. After excluding aberrant samples
by probe quality control, Principal Components Analysis
(PCA) and clustering, 345 patients' data including 345
tumor tissues and 50 adjacent normal tissues were
obtained. Totally, 246 HCC samples and their matched
adjacent normal tissues from five datasets were eligible
for meta-analysis. Only TCGA dataset was used for
survival analyses.

\section{Meta-analysis}

A meta-analysis approach was applied to the normalized data. It combines effect sizes from each dataset into a meta-effect size to estimate the amount of change in expression across all datasets. For the site of the gene in each dataset, an effect size was computed using Hedges' adjusted. The study-specific effect sizes were combined to obtain the pooled effect size and its standard error using the random effects inverse-variance technique. The Z-statistic was computed as a ratio of the pooled effect size to its standard error for the gene, and the result was compared to a standard normal distribution to obtain a nominal $p$-value.

\section{Pyrosequencing analysis of DNA methylation}

The primers were designed using Pyrosequencing ${ }^{\mathrm{TM}}$ Assay Design Software Version 1.0; where one of the primers was biotin-labeled. Primer sequences were as follows: Sense primer biotin-5'-GGAA GGTGTTGGAGGATATT-3', antisense primer 5'-CCT CTCAACCCTACATATCTCTTATCA-3', sequencing primer 5'-ATCTCTTATCAACTATCTTTCA-3'. The Platinum PCR SurperMix High Fidelity (Invitrogen, Carlsbad, CA) was used to prepare the PCR reaction solution. After the biotinylated strand was captured on streptavidin-coated beads (Amersham Bioscience, Uppsala, Sweden) and incubated with sequencing primers, the specific PCR products were then subjected to quantitative pyrosequencing analysis using a Pyrosequencing ${ }^{\mathrm{TM}}$ PyroMark MD system following the protocol provided by the manufacturer. The sequencing results were analyzed using the PyroMark Q96 software (Qiagen).

\section{Construction of S100A8 lentiviral vectors}

To investigate the effect of S100A8 overexpression on HCC cell lines, S100A8 (NM_002964.4) lentiviral vector with a puromycin selection marker was constructed (Genecopoeia Co. Ltd., Guangzhou, China), and empty vectors were used as controls.

\section{Western blot analysis}

Equal amounts of total proteins $(20 \mu \mathrm{g})$ were separated by $10 \%$ SDS-PAGE and transferred onto PVDF membrane using a Bio-Rad SemiDry apparatus. The membrane was blocked by $5 \%$ milk or $2 \%$ BSA at room temperature for 1 $\mathrm{h}$. Then, the membrane was incubated with specific primary antibody with suitable dilution at $4^{\circ} \mathrm{C}$ overnight. After 3 times of 10 min washing by TBST, the membrane was further incubated with HRP-conjugated secondary antibodies (Bio$\mathrm{Rad}$ ) at room temperature for $1 \mathrm{~h}$, and then washed again by TBST for 3 times of $10 \mathrm{~min}$. ECL Western Blotting Detection Reagents (Bio-Rad) and ChemiDoc XRS ${ }^{+}$system (Bio-Rad) were used to visualize the bands on membrane. 


\section{Cell proliferation assays}

Huh7 cells and MHCC-97H cells (1000 cells/well) were dispensed in $100 \mu \mathrm{L}$ aliquots into a 96-well plate. At the indicated time points, the 2-(4-indophenyl)-3-(4-nitrophenyl)5-(2,4-disulphophenyl)-2H-tetrazolium monosodium salt (CCK8, Cell Counting kit) was added to the cells for $1 \mathrm{~h}$, and then the plate was read using an enzyme-linked immunosorbent assay plate reader at $450 \mathrm{~nm}$.

\section{Scratch-wound assay}

Seed cells into 24-well tissue culture plate at a density that after $24 \mathrm{~h}$ of growth, they should reach $70-80 \%$ confluence as a monolayer. Gently and slowly scratch the monolayer with a new $200 \mu \mathrm{l}$ pipette tip across the center of the well. Scratch a straight line in one direction. Scratch another straight line perpendicular to the first line to create a cross in each well. After 48 hours incubation at $37{ }^{\circ} \mathrm{C}$, take photos on a microscope. The gap distance can be quantitatively evaluated using Photoshop. Each experimental group should be repeated 3 times.

\section{Cell invasion Assay}

For in vitro invasion assay, transwells coated with Matrigel (Millipore) were utilized according to manufacturer's instructions. $5 \times 10^{4}$ cells in $100 \mu \mathrm{l}$ serumfree DMEM were seeded in the upper chamber of a transwell and $500 \mu \mathrm{l}$ medium contain $15 \% \mathrm{FBS}$ was added to the lower chamber. After 30 hours incubation at $37^{\circ} \mathrm{C}$, invaded cells were fixed with $20 \%$ methanol and stained with Hematoxylin Staining Solution. Cells adhering to the lower side of the inserts were counted and imaged through microscope. Five random microscopic fields were counted per well for each group, and the experiments were repeated at least three times independently.

\section{Tumor formation assay}

Male BALB/C nude mice (5-6 week old) were obtained from Shanghai Institute of Materia Medica (Chinese Academy of Sciences, Shanghai, China). The in vivo experiments were carried out strictly in accordance with a protocol approved by the Guangxi Medical University Experimental Animal Care Committee. $1 \times 10^{7}$ cells were injected subcutaneously into the upper left flank region of nude mice. The mice were sacrificed at the 28th day after cell injection.

\section{Statistical analysis}

Statistical analyses were performed using the SPSS 16.0 software package. Data were expressed as mean \pm SD. One-way ANOVA was employed to determine the differences among different groups. Pearson partial correlation analysis was used to analyze the correlation of multiple variables. Receiver operating characteristic (ROC) curves were used to determine the diagnostic value of the markers. The best cut-off value for each protein was defined as the point with maximum Youden index (sensitivity+ specificity -1) on the ROC curve. Survival curves were generated using the Kaplan-Meier method, and comparisons between the curves were made using the log-rank test. Patients were divided into four quartiles based on methylation level of tumor sample. Overall survival (OS) and progression-free survival (PFS) were compared between the quartiles. OS was taken from "days to death" for deceased patients and "days_to_last_followup" for patients reported living. PFS was taken from patients with reported "days to new tumor event", and PFS equal to OS for blank or "NA" entries. Multivariate survival analyses were performed with the Cox proportional hazards model. The reference groups were 'high-methylation-level group'. $p<0.05$ was considered statistically significant.

\section{CONFLICTS OF INTEREST}

The authors declare that they have no conflicts of interest in the research

\section{REFERENCES}

1. Kimhofer T, Fye H, Taylor-Robinson S, Thursz M, Holmes E. Proteomic and metabonomic biomarkers for hepatocellular carcinoma: a comprehensive review. British journal of cancer. 2015;112:1141-56.

2. Singal AG, El-Serag HB. Hepatocellular Carcinoma From Epidemiology to Prevention: Translating Knowledge into Practice. Clinical gastroenterology and hepatology. 2015;13:2140-51.

3. Yan X, Qiu Y. Impact of current staging systems on treatment strategy for HBV-related hepatocellular carcinoma. Cancer Lett. 2016; 379:220-4.

4. Attwa MH, El-Etreby SA. Guide for diagnosis and treatment of hepatocellular carcinoma. World journal of hepatology. 2015;7:1632-51.

5. Gnoni A, Santini D, Scartozzi M, Russo A, Licchetta A, Palmieri V, Lupo L, Faloppi L, Palasciano G, Memeo V, Angarano G, Brunetti O, Guarini A, et al. Hepatocellular carcinoma treatment over sorafenib: epigenetics, microRNAs and microenvironment. Is there a light at the end of the tunnel? Expert opinion on therapeutic targets. 2015:1-13.

6. Kanda T, Ogasawara S, Chiba T, Haga Y, Omata M, Yokosuka O. Current management of patients with hepatocellular carcinoma. World journal of hepatology. 2015;7:1913-20.

7. Schlachterman A, Craft WW, Jr., Hilgenfeldt E, Mitra A, Cabrera R. Current and future treatments for 
hepatocellular carcinoma. World journal of gastroenterology. 2015;21:8478-91.

8. Oprita R, Diaconescu IB, Lupu G, Lupu A, Cristea B, Bratu MR. Hepatocellular carcinoma among cirrhotics--utility of screening and surveillance programs--review article. Journal of medicine and life. 2014;7:477-80.

9. Fan H, Zhao H, Pang L, Liu L, Zhang G, Yu F, Liu T, Xu C, Xiao Y, Li X. Systematically Prioritizing Functional Differentially Methylated Regions (fDMRs) by Integrating Multi-omics Data in Colorectal Cancer. Scientific reports. 2015;5:12789.

10. Fan H, Zhao Z, Cheng Y, Cui H, Qiao F, Wang L, Hu J, Wu $\mathrm{H}$, Song W. Genome-wide profiling of DNA methylation reveals preferred sequences of DNMTs in hepatocellular carcinoma cells. Tumour Biol. 2016; 37:877-85.

11. Merry CR, Forrest ME, Sabers JN, Beard L, Gao XH, Hatzoglou M, Jackson MW, Wang Z, Markowitz S, Khalil AM. DNMT1-associated long non-coding RNAs regulate global gene expression and DNA methylation in colon cancer. Human molecular genetics. 2015.

12. Pouliot MC, Labrie Y, Diorio C, Durocher F. The Role of Methylation in Breast Cancer Susceptibility and Treatment. Anticancer research. 2015;35:4569-74.

13. Anzola M, Cuevas N, Lopez-Martinez M, Martinez de Pancorbo M, Burgos JJ. p16INK4A gene alterations are not a prognostic indicator for survival in patients with hepatocellular carcinoma undergoing curative hepatectomy. Journal of gastroenterology and hepatology. 2004;19:397-405.

14. Herath NI, Leggett BA, MacDonald GA. Review of genetic and epigenetic alterations in hepatocarcinogenesis. Journal of gastroenterology and hepatology. 2006;21:15-21.

15. Harada K, Baba Y, Ishimoto T, Chikamoto A, Kosumi K, Hayashi H, Nitta H, Hashimoto D, Beppu T, Baba H. LINE-1 methylation level and patient prognosis in a database of 208 hepatocellular carcinomas. Annals of surgical oncology. 2015;22:1280-7.

16. Zamani M, Sadeghizadeh M, Behmanesh M, Najafi F. Dendrosomal curcumin increases expression of the long non-coding RNA gene MEG3 via up-regulation of epi-miRs in hepatocellular cancer. Phytomedicine. 2015;22:961-7.

17. Frau M, Feo F, Pascale RM. Pleiotropic effects of methionine adenosyltransferases deregulation as determinants of liver cancer progression and prognosis. Journal of hepatology. 2013;59:830-41.

18. Okada H, Kimura MT, Tan D, Fujiwara K, Igarashi J, Makuuchi M, Hui AM, Tsurumaru M, Nagase H. Frequent trefoil factor 3 (TFF3) overexpression and promoter hypomethylation in mouse and human hepatocellular carcinomas. International journal of oncology. 2005;26:369-77.

19. Xu YD, Yin LM, Wang Y, Wei Y, Yang YQ. [S100A8 protein in inflammation]. [Article in Chinese]. Sheng li xue bao. 2012;64:231-7.
20. Bresnick AR, Weber DJ, Zimmer DB. S100 proteins in cancer. Nature reviews Cancer. 2015;15:96-109.

21. Gebhardt C, Breitenbach U, Tuckermann JP, Dittrich BT, Richter KH, Angel P. Calgranulins S100A8 and S100A9 are negatively regulated by glucocorticoids in a c-Fosdependent manner and overexpressed throughout skin carcinogenesis. Oncogene. 2002;21:4266-76.

22. Lesniak W. Epigenetic regulation of S100 protein expression. Clinical epigenetics. 2011;2:77-83.

23. Saeki N, Ono H, Sakamoto H, Yoshida T. Down-regulation of Immune-related Genes by PSCA in Gallbladder Cancer Cells Implanted into Mice. Anticancer research. 2015;35:2619-25.

24. Funk S, Mark R, Bayo P, Flechtenmacher C, Grabe N, Angel P, Plinkert PK, Hess J. High S100A8 and S100A12 protein expression is a favorable prognostic factor for survival of oropharyngeal squamous cell carcinoma. International journal of cancer. 2015;136:2037-46.

25. Reeb AN, Li W, Sewell W, Marlow LA, Tun HW, Smallridge RC, Copland JA, Spradling K, Chernock R, Lin RY. S100A8 is a novel therapeutic target for anaplastic thyroid carcinoma. The Journal of clinical endocrinology and metabolism. 2015;100:E232-42.

26. Silva EJ, Argyris PP, Zou X, Ross KF, Herzberg MC. S100A8/A9 regulates MMP-2 expression and invasion and migration by carcinoma cells. The international journal of biochemistry \& cell biology. 2014;55:279-87.

27. Basso D, Bozzato D, Padoan A, Moz S, Zambon CF, Fogar P, Greco E, Scorzeto M, Simonato F, Navaglia F, Fassan M, Pelloso M, Dupont S, et al. Inflammation and pancreatic cancer: molecular and functional interactions between S100A8, S100A9, NT-S100A8 and TGFbeta1. Cell communication and signaling. 2014;12:20.

28. Jou YJ, Hua CH, Lin CD, Lai CH, Huang SH, Tsai MH, Kao JY, Lin CW. S100A8 as potential salivary biomarker of oral squamous cell carcinoma using nanoLC-MS/MS. Clinica chimica acta. 2014;436:121-9.

29. Mirza Z, Schulten HJ, Farsi HM, Al-Maghrabi JA, Gari MA, Chaudhary AG, Abuzenadah AM, Al-Qahtani MH, Karim S. Impact of S100A8 expression on kidney cancer progression and molecular docking studies for kidney cancer therapeutics. Anticancer research. 2014;34:1873-84.

30. Yang M, Zeng P, Kang R, Yu Y, Yang L, Tang D, Cao L. S100A8 contributes to drug resistance by promoting autophagy in leukemia cells. PloS one. 2014;9:e97242.

31. Khammanivong A, Sorenson BS, Ross KF, Dickerson EB, Hasina R, Lingen MW, Herzberg MC. Involvement of calprotectin (S100A8/A9) in molecular pathways associated with HNSCC. Oncotarget. 2016; 7:14029-47. doi: 10.18632/oncotarget.7373.

32. Kessels JE, Wessels I, Haase H, Rink L, Uciechowski P. Influence of DNA-methylation on zinc homeostasis in myeloid cells: Regulation of zinc transporters and zinc binding proteins. Journal of trace elements in medicine and biology. 2016. 
33. Chang H, Yi B, Li L, Zhang HY, Sun F, Dong SQ, Cao Y. Methylation of tumor associated genes in tissue and plasma samples from liver disease patients. Experimental and molecular pathology. 2008;85:96-100.

34. Harder J, Opitz OG, Brabender J, Olschewski M, Blum HE, Nomoto S, Usadel H. Quantitative promoter methylation analysis of hepatocellular carcinoma, cirrhotic and normal liver. International journal of cancer. 2008;122:2800-4.

35. He C, Xu J, Zhang J, Xie D, Ye H, Xiao Z, Cai M, Xu K, Zeng Y, Li H, Wang J. High expression of trimethylated histone H3 lysine 4 is associated with poor prognosis in hepatocellular carcinoma. Human pathology. 2012;43:1425-35.

36. Wong IH, Lo YM, Zhang J, Liew CT, Ng MH, Wong N, Lai PB, Lau WY, Hjelm NM, Johnson PJ. Detection of aberrant p16 methylation in the plasma and serum of liver cancer patients. Cancer research. 1999;59:71-3.

37. Yuan SX, Zhang J, Xu QG, Yang Y, Zhou WP. Long noncoding RNA, the methylation of genomic elements and their emerging crosstalk in hepatocellular carcinoma. Cancer Lett. 2015.

38. ZHANG Y-s, SHI Y-1, XIAO D-y. Application of ROC Curve in the Analysis and Evaluation of the Diagnostic Value of the Combined Detection of CYFRA21-1 and CEA in Lung Cancer [J]. Journal of Clinical Research. 2007;7:012.

39. Alsina AE, Nakshabandi A, Makris AM, Torres EA. Liver transplantation for hepatocellular carcinoma in Puerto Ricans: underutilization of a curative therapy. Puerto Rico health sciences journal. 2014;33:170-6.
40. Colombo M, Sangiovanni A. Treatment of hepatocellular carcinoma: beyond international guidelines. Liver international. 2015;35:129-38.

41. Schlageter M, Terracciano LM, D'Angelo S, Sorrentino P. Histopathology of hepatocellular carcinoma. World journal of gastroenterology. 2014;20:15955-64.

42. De Ponti A, Wiechert L, Schneller D, Pusterla T, Longerich T, Hogg N, Vogel A, Schirmacher P, Hess J, Angel P. A pro-tumorigenic function of S100A8/A9 in carcinogeninduced hepatocellular carcinoma. Cancer letters. 2015;369:396-404.

43. Duan L, Wu R, Ye L, Wang H, Yang X, Zhang Y, Chen X, Zuo G, Zhang Y, Weng Y, Luo J, Tang M, Shi Q, et al. S100A8 and S100A9 are associated with colorectal carcinoma progression and contribute to colorectal carcinoma cell survival and migration via Wnt/beta-catenin pathway. PloS one. 2013;8:e62092.

44. Yang XL, Zhang CD, Wu HY, Wu YH, Zhang YN, Qin MB, Wu H, Liu XC, Lina X, Lu SM. Effect of trichostatin A on CNE2 nasopharyngeal carcinoma cells--genome-wide DNA methylation alteration. Asian Pacific journal of cancer prevention. 2014;15:4663-70.

45. Teschendorff AE, Marabita F, Lechner M, Bartlett T, Tegner J, Gomez-Cabrero D, Beck S. A beta-mixture quantile normalization method for correcting probe design bias in Illumina Infinium $450 \mathrm{k}$ DNA methylation data. Bioinformatics (Oxford, England). 2013;29:189-96. 